\title{
Pharmacotherapy of pediatric and adolescent HIV infection
}

This article was published in the following Dove Press journal:

Therapeutics and Clinical Risk Management

17 June 2009

Number of times this article has been viewed

\author{
Susan J Schuval ${ }^{1,2}$ \\ 'Division of Allergy/Immunology, \\ Department of Pediatrics, North \\ Shore - Long Island Jewish Health \\ System, Great Neck, NY, USA; \\ ${ }^{2}$ Associate Professor of Clinical \\ Pediatrics, Albert Einstein College \\ of Medicine, Bronx, NY, USA
}

Correspondence: Susan J Schuval Division of Allergy/lmmunology, 865 Northern Boulevard, Suite I0I, Schneider Children's Hospital, Great Neck, NY II02I, USA

Tel +I 5166225070

Fax +I 5166225060

Email schuval@lij.edu
Abstract: Significant advances have been made in the treatment of human immunodeficiency virus (HIV) infection over the past two decades. Improved therapy has prolonged survival and improved clinical outcome for HIV-infected children and adults. Sixteen antiretroviral (ART) medications have been approved for use in pediatric HIV infection. The Department of Health and Human Services (DHHS) has issued "Guidelines for the Use of Antiretroviral Agents in Pediatric HIV Infection”, which provide detailed information on currently recommended antiretroviral therapies (ART). However, consultation with an HIV specialist is recommended as the current therapy of pediatric HIV therapy is complex and rapidly evolving.

Keywords: antiretroviral, HAART, Pediatric Guidelines, HIV, AIDS

\section{Introduction}

The first cases of pediatric Acquired Immunodeficiency Syndrome (AIDS) were described in the United States (US) in 1983. ${ }^{1}$ At that time, no specific antiretroviral (ARV) medications were available to children infected with HIV, most of whom succumbed to their infection. In 1990, zidovudine became the first approved pediatric ARV medication. HIV-infected children received zidovudine monotherapy with some clinical improvement but HIV infection remained a fatal disease. However, with the evolution of highly active antiretroviral therapy (HAART) during the 1990's, HIV-associated morbidity and mortality declined greatly, and HIV infection became a treatable and chronic disease for both adults and children. At the present time, most HIV-infected children in the US are surviving through adolescence to adulthood. In the US, most children acquire HIV via perinatal transmission, although infection may be acquired behaviorally (sexually or via injection drug use) in adolescents.

Currently, there is no cure for HIV infection and viral eradication is not possible at this time. However, viral suppression to undetectable levels can be achieved by individualization of therapy, with careful selection of initial and subsequent treatment regimens. Maximal viral suppression is associated with delayed HIV disease progression and improved survival. ${ }^{2}$ Maintaining adherence to prescribed regimens, preventing short-term and long-term drug toxicities, and managing drugresistance in HIV-infected children and adolescents receiving ART are the main challenges faced today in the treatment of pediatric HIV infection. Although many of the medications used for treatment are the same as those used in adults, issues unique to pediatric HIV infection include differences in mode of viral acquisition, immaturity of the immune system, and considerations of growth and development, 
especially during puberty. Treatment decisions must include consideration of the child's age, treatment readiness, psychosocial milieu, past treatment history, as well as available drug formulations and treatment schedule. Possible drug teratogenicity must be considered in the treatment of adolescents who may be sexually active. Goals of ART include reduction of HIV-associated morbidity and mortality, preservation or restoration of immune function, and reduction of HIV viral replication. Whenever possible, consultation with a specialist in the treatment of pediatric or adolescent HIV infection is recommended as the current therapy of pediatric HIV infection is complex and rapidly evolving.

This review summarizes the current "Guidelines for the Use of Antiretroviral Agents in Pediatric HIV Infection."3 The ARV medications discussed below apply primarily to highly resourced settings such as the US. Although zidovudine given perinatally to mother and child has been proven to successfully interrupt perinatal HIV transmission, use of post-exposure prophylaxis will not be discussed here nor will prophylaxis and treatment of opportunistic infections in HIV-infected children. ${ }^{4,5}$

\section{HIV virology/Life cycle}

HIV-1 is a human retrovirus which contains the enzyme reverse transcriptase. During acute HIV infection, the viral envelope glycoprotein gp 120 binds to the CD4 receptor on the mature human helper T lymphocyte (CD4 cell). Subsequently, the viral envelope binds to 1 of 2 chemokine coreceptors, either CXCR4 (X4) or CCR5 (R5). Most viral isolates acquired via sexual transmission utilize the R5 chemokine receptor (R5-tropic) while X4 viral strains tend to predominate in more advanced HIV infection (X4-tropic). A conformational change then ensues, resulting in fusion of the viral envelope with the CD4 cell membrane, after which the HIV viral RNA enters the cell cytoplasm. ${ }^{6}$ The viral reverse transcriptase then transcribes the singlestranded viral RNA into host double stranded DNA. The viral enzyme integrase inserts the proviral DNA into the host genome via a strand transfer reaction. During subsequent activation of this infected CD4 T cell, the integrated proviral DNA is translated into new viral proteins. Assembly of these viral products then occurs on the cell surface, followed by budding of immature viral particles. The viral enzyme protease aids in the production of final infectious virions by cleaving viral polyprotein precursors, and generating new viral proteins. Active viral replication then occurs, eventually decreasing over time to a steady state level. A progressive immunodeficiency is seen over time, which may culminate in the occurrence of opportunistic infections, malignancy, and death.

\section{Overview of antiretroviral medications}

Since 1996, 31 ARVs have been approved for use, with 16 of these approved for use in children (Table 1). Three of these medications (zalcitabine, amprenavir, and delavirdine) are no longer manufactured. There are currently six classes of medications for treatment of HIV infection that block various stages of the HIV life cycle by targeting key enzymes. ARVs such as reverse transcriptase inhibitors (both nucleoside reverse transcriptase inhibitors and non-nucleoside reverse transcriptase inhibitors), integrase inhibitors, and protease inhibitors prevent replication of the viral genome and intracellular viral maturation. Fusion inhibitors and CCR5 antagonists block the entry of virus into the CD4 cell either by interfering with viral fusion or co-receptor binding. Nucleoside reverse transcriptase inhibitors, non-nucleoside reverse transcriptase inhibitors, and protease inhibitors all have pediatric indications, while entry inhibitors, CCR5 antagonist, and integrase inhibitors are newer agents used primarily for the treatment of multidrug-resistant HIV infection in adults. Pediatric experience with these newer medications is limited and their use is only currently recommended in cases of treatment failure. Most of the data regarding pediatric HIV treatment are derived from adult data or Phase I/II clinical trials as few phase III ART clinical trials have been performed in HIV-infected children. However, HAART regimens have been shown to be safe efficacious, and durable in HIV-infected children, even with longterm use..$^{7-9}$

The following will serve as an overview of the medications currently used in the treatment of HIV-infected children and adolescents. More detailed information on each medication can be found elsewhere. ${ }^{3}$

\section{Classes of antiretroviral medications \\ Nucleoside reverse transcriptase inhibitors (NRTIs)}

NRTIs were the first class of ARV medications approved for the treatment of HIV infection. NRTIs inhibit the viral enzyme reverse transcriptase, preventing transcription of viral RNA into host DNA. These nucleoside analogues are prodrugs that require intracellular phosphorylation for activation. The 6 NRTIs currently approved for use in HIV-infected children 


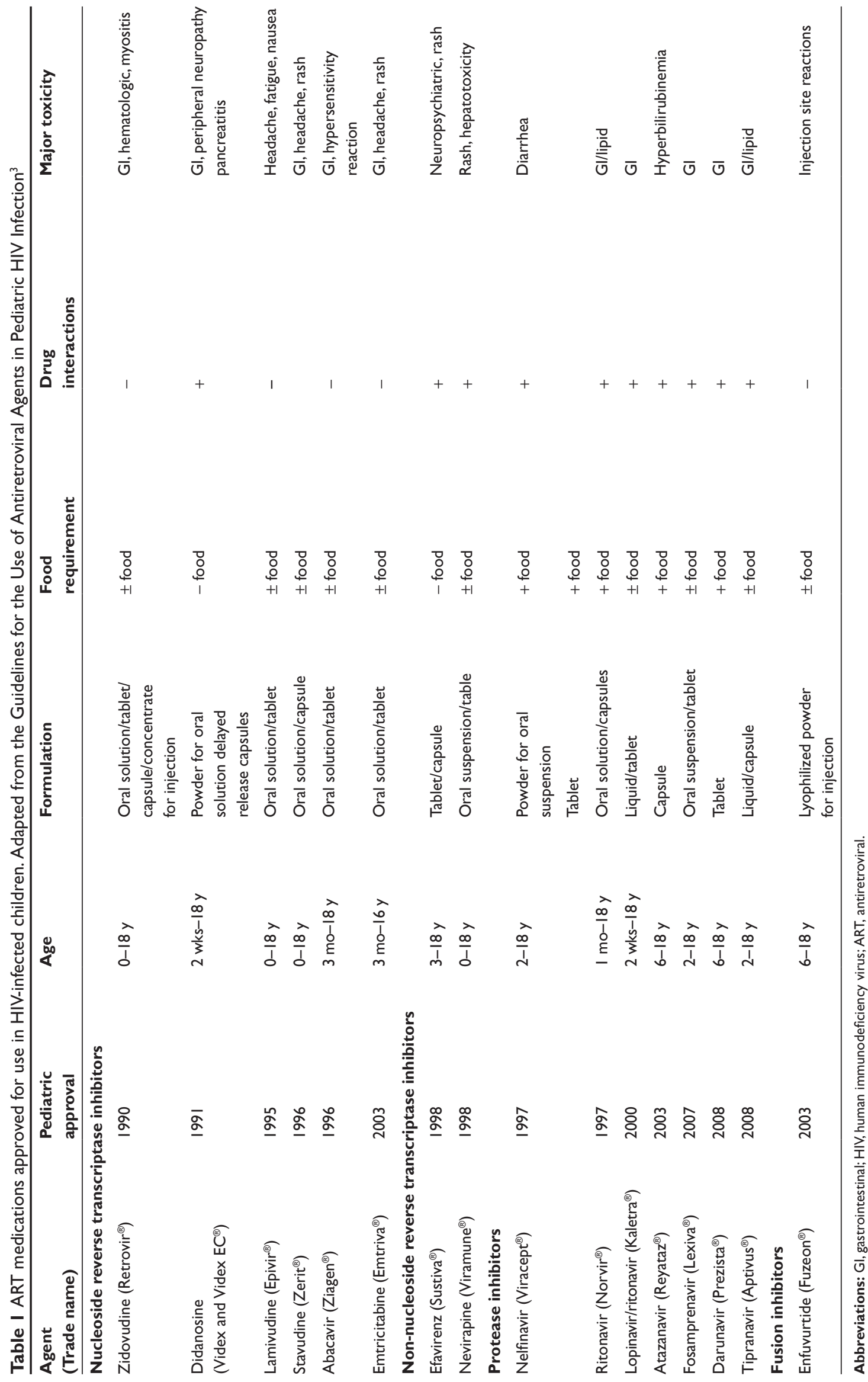


include zidovudine, stavudine, lamivudine, emtricitabine, didanosine, and abacavir (Table 1). NRTIs are currently recommended for use in all pediatric ART regimens as part of a dual NRTI backbone. ${ }^{3}$ NRTIs have few side effects and are mostly well-tolerated with good bioavailability. NRTIs such as zidovudine penetrate into cerebrospinal fluid and may be useful in the treatment of HIV-associated neurocognitive disorders. NRTIs have few drug interactions and are mostly eliminated via the kidneys, necessitating possible dose adjustment in renal failure. Use of NRTIs has been linked with mitochondrial toxicity, which may present as cardiomyopathy, myopathy, peripheral neuropathy, pancreatitis, renal tubular dysfunction, hepatic steatosis, and lactic acidosis, all of which occur more often in adults than children.

\section{Thymidine analogues}

\section{Zidovudine (Retrovir ${ }^{\circledR}$ )}

Zidovudine was the first ARV medication ever approved. It is available in liquid, capsule, tablet, and a concentrate form for injection or intravenous infusion. The oral form is usually administered twice daily (thrice daily in younger children), with the dose determined by weight or body surface area. Although it is generally well-tolerated by children, bone marrow suppression may occur, resulting in anemia and leukopenia. Other common side effects include headache, malaise, and gastrointestinal symptoms such as nausea, vomiting, and anorexia. Myopathy, myositis, liver toxicity, and fat redistribution are less common side effects. ${ }^{10,11}$

\section{Stavudine $\left(\right.$ Zerit $\left.^{\circledR}\right)$}

Stavudine is available as a capsule or solution for twice daily administration. Common toxicities include headache, rash, and gastrointestinal symptoms. Less common side effects include peripheral neuropathy, lipodystrophy, serum transaminase elevation, and neuromuscular weakness. Although use of all NRTIs has been linked to lipoatrophy and hyperlactatemia, this effect is seen more often with stavudine. ${ }^{12}$ Thus, at the present time, use of stavudine is limited to special circumstances (such as a young child with human leukocyte antigen [HLA] B*5701 who cannot be given abacavir, or a child with anemia who may experience additional hematologic suppression from zidovudine). ${ }^{3}$

\section{Nonthymidine analogues}

Lamivudine (Epivir ${ }^{\circledR}$ )

Lamivudine is a cytosine analogue that has been extensively studied in HIV-infected children. ${ }^{11,13}$ It is available both as an oral solution and a tablet, that are well-tolerated with few side effects (headache, fatigue, anorexia, rash, abdominal pain, and insomnia). Less common side effects include pancreatitis, peripheral neuropathy, anemia, neutropenia, serum transaminase elevation, and fat redistribution.

\section{Emtricitabine (Emtriva ${ }^{\circledR}$ )}

Emtricitabine is a pyrimidine nucleoside analogue that is a fluorinated derivative of lamivudine. It was approved in 2003 for once daily dosing and is available in a palatable liquid formulation. Emtricitabine was shown to be safe, well tolerated, and durable in the treatment of HIV-infected children as young as three months. ${ }^{14}$ Its longer half-life, higher oral bioavailability, and slightly greater potency in vitro led to its inclusion in combination with zidovudine over lamivudine as a preferred component of the dual NRTI backbone. ${ }^{3}$ It may be taken with or without food, and has few drug interactions. Side effects are generally mild and include rash, headache, insomnia, diarrhea, nausea, and vomiting. Less commonly seen adverse events include neutropenia and skin hyperpigmentation which has been reported in African-American patients receiving emtricitabine.

\section{Didanosine (Videx and Videx EC ${ }^{\circledR}$ )}

Didanosine is an adenosine analogue that is available as a delayed-release capsule for once daily administration or a pediatric powder for oral solution. Although didanosine is best absorbed on an empty stomach, pediatric pharmacokinetic studies have shown that acceptable drug levels are achieved, although with delayed and slower absorption in children, when it is administered with food. ${ }^{15}$ The tablets and the oral solution contain antacids which may affect the absorption of other medications. Didanosine's side effects are predominantly gastrointestinal, consisting of nausea, vomiting, diarrhea, and abdominal pain. Less commonly seen are peripheral neuropathy, electrolyte abnormalities and hyperuricemia. Pancreatitis, retinal changes, hepatotoxicity, and optic neuritis have also been reported. The virologic efficacy of combined didanosine, emtricitabine, and efavirenz has been proven in pediatric trials, resulting in its selection as a preferred dual NRTI agent in combination with emtricitabine. , $^{3,16}$

\section{Abacavir (Ziagen ${ }^{\circledR}$ )}

Abacavir is a guanosine analogue that has been shown to be safe and efficacious for long-term use in HIV-infected children. ${ }^{13,17}$ The most common side effects associated with its use include nausea, vomiting, diarrhea, anorexia, fever, headache, and rash. ${ }^{18}$ Although abacavir is a potent 
suppressor of viral replication, it has the potential to cause a life-threatening hypersensitivity reaction in genetically predisposed individuals who possess the HLA allele B*5701. This hypersensitivity reaction occurs in 5\%-8\% of recipients, and consists of fever, rash, systemic, gastrointestinal and respiratory symptoms, usually during the first 6 weeks of therapy. Continuation of abacavir therapy has been associated with increased severity of symptoms, and fatality. Rechallenge with abacavir is contraindicated in anyone reporting past hypersensitivity reaction. Pharmacogenetic testing for the HLA phenotype B*5701 is recommended prior to use in HIV-infected patients, and abacavir should be withheld from those who test positive for this allele. HIV-infected children and adults receiving abacavir should be informed of this risk of hypersensitivity reaction, and instructed to immediately contact their provider regarding abacavir discontinuation, if fever and rash occur. ${ }^{19}$ Other side effects of abacavir include nausea, diarrhea, abdominal pain, asthenia, headache, and elevation of serum transaminases or creatinine.

\section{Nucleotide analogues}

Tenofovir disoproxil fumarate (Viread ${ }^{\circledR}$ )

Tenofovir disoproxil fumarate is a nucleotide analogue of deoxyadenosine monophosphate approved for use in young adults $>18$ years. Its long half life allows for once daily administration. It is available only in tablet form, and may be taken with or without food, although its absorption is enhanced with food. Tenofovir is excreted unchanged by the kidneys; renal tubular toxicity is a rare side effect of therapy. However, monitoring of renal function is recommended during tenofovir therapy, and dose adjustment is necessary in renal failure. Other side effects include nausea, diarrhea, rash, and flatulence. Lack of pediatric dosing information and possible bone toxicity preclude the use of tenofovir in prepubertal children (Tanner Stages $1-3$ ) or those $\leq 18$ years. ${ }^{20}$ However use of tenofovir may be considered in older postpubertal children. ${ }^{3}$ Drug interactions with numerous ARVs are seen, and dose adjustment may be necessary. Tenofovir increases serum concentrations of didanosine, requiring decreased didanosine dose, while dose increases are necessary for tipranavir, since tenofovir decreases serum concentrations of this medication. Concomitant lopinavir/ritonavir may increase serum tenofovir levels, possibly resulting in greater tenofovir toxicity. ${ }^{21}$ Tenofovir also decreases serum levels of atazanavir. If tenofovir and atazanavir are co-administered, ritonavir boosting should be used with the atazanavir; use of unboosted atazanavir with tenofovir is not recommended.

\section{Non-nucleoside reverse transcriptase inhibitors (NNRTIs)}

The NNRTIs also inhibit the enzyme reverse transcriptase. By binding noncompetitively to the hydrophobic pocket close to the polymerase catalytic site of the reverse transcriptase, NNRTIs decrease reverse transcriptase polymerizing activity. NNRTIs include nevirapine, efavirenz, delavirdine, and etravirine, but only efavirenz and nevirapine have pediatric indications. Although NNRTIs have low pill burdens and cause less hyperlipidemia and fat maldistribution, the rapid emergence of class resistance, and high frequency of cutaneous reactions associated with these medications are drawbacks to their use. Both efavirenz and nevirapine have very long half-lives and may remain detectable for up to 21 days after discontinuation. Most NNRTIs are metabolized by the liver and drug interactions are possible with this class of medications. ${ }^{3}$

\section{Efavirenz (Sustiva ${ }^{\circledR}$ )}

Efavirenz is the preferred NNRTI in children due to its low toxicity and once daily administration. However, it is formulated only as a capsule and approved only for children $\geq 3$ years weighing $\geq 10 \mathrm{~kg}$. Its absorption is enhanced on an empty stomach. Dosing for efavirenz is usually weight-based, but body surface area dosing has also been utilized, especially in regimens containing lopinavir/ ritonavir. ${ }^{22,23}$ Efavirenz is well-tolerated, but neuropsychiatric side effects such as dizziness, fatigue, insomnia, agitation, impaired concentration, vivid dreams, and suicidal ideation may occur with its use. For this reason, it should be administered at bedtime, and cautious use is suggested in patients with psychiatric disease. Rash occurs less commonly than with nevirapine, but lipid abnormalities have been reported Efavirenz is teratogenic and its use should be avoided in women of reproductive age unless adequate contraception is ensured. Efavirenz is a mixed inducer/inhibitor of cytochrome P450 3A4 enzymes and is associated with many drug interactions. It may reduce levels of lopinavir, saquinavir, and indinavir, if co-administered with these drugs. Concomitant administration of midazolam and triazolam should be avoided. Studies have demonstrated the virologic efficacy of efavirenz in combination with NRTIs and/or PIs in the treatment of pediatric HIV infection. ${ }^{16,24-26}$

\section{Nevirapine (Viramune ${ }^{\circledR}$ )}

Nevirapine was the first NNRTI approved by the US Food and Drug Administration (FDA). It is available both as a tablet and a liquid formulation, both of which may be given with or 
without food. Nevirapine should be used in children $\leq 3$ years, or in children requiring a liquid NNRTI formulation, if an NNRTI-based regimen is desired. It has been proven safe and efficacious in pediatric clinical trials. ${ }^{27,28}$ The most common side effect is rash, seen in $5 \%$ of treated children, usually during the second week of therapy. ${ }^{10}$ Erythema multiforme, Stevens-Johnson syndrome, and toxic epidermal necrolysis have been reported; nevirapine should be discontinued in the case of a severe rash; milder skin eruptions generally resolve within 1-2 weeks and do not require drug discontinuation. Initiation of half-dose nevirapine therapy, followed by stepwise dose escalation over a two-week period allows for autoinduction of cytochrome p450 metabolizing enzymes CYP3A and 2B6, resulting in increased drug clearance and decreased cutaneous toxicity. Although, life-threatening hepatotoxicity has also been reported in adults with higher CD4 counts, usually during the first 12 weeks of therapy, nevirapine-induced hepatotoxicity is seen infrequently in children. ${ }^{29}$ Concomitant use of other potentially hepatotoxic medications should be avoided. Serum transaminase levels should be checked every two weeks during the first month of nevirapine therapy, then monthly for three months, and then every three to four months. Because of potential hepatotoxicity, nevirapine should not be used in post-pubertal females with CD4 $>250$ or males with CD4 $>400$, unless benefits of therapy outweigh the risks. ${ }^{30,31}$ Nevirapine also has the potential for numerous drug interactions as it acts as an inducer of hepatic cytochrome p450 enzymes including CYP3A and 2B6. Certain PI doses may require adjustment if given with nevirapine.

Two other NNRTIs have been approved for HIV-infected adults but not children. These include Delavirdine (Rescriptor) and Etravirine (Intelence). Delavirdine is no longer manufactured Etravirine is a second generation NNRTI active against viruses with resistance to first generation NNRTIs that is useful in treatment-experienced adults. However, this medication has not been adequately studied in children and cannot be recommended at the present time. ${ }^{3}$

\section{Protease inhibitors}

There are seven protease inhibitors (PIs) currently approved for use in children. The use of combination therapy with PIs in HIV-infected children and adolescents has been shown to reduce mortality by $67 \% .{ }^{32}$ PIs inhibit the viral enzyme protease by binding competitively to the enzyme, limiting the production of mature infectious virions. PIs have a high genetic barrier for the development of drug resistance and are potent inhibitors of viral replication. However, their use is complicated by high pill burdens, numerous drug interactions, and frequent metabolic side effects. Abnormalities of glucose metabolism (hyperglycemia, insulin resistance, or diabetes mellitus) and changes in body fat distribution (lipodystrophy) and dyslipidemias (hypertriglyceridemia, hypercholesterolemia) are commonly attributed to this class of medications. In addition, PIs have been associated with serum transaminase elevation, hepatitis, pancreatitis, and spontaneous bleeding in hemophiliacs. Unpalatable drug formulations and large pill size are drawbacks to PI use. Also, drug interactions occur frequently with this class of medications that are metabolized by the hepatic cytochrome P450 enzyme system, necessitating possible dose adjustments. For example, decreased levels of rifabutin, rifampin, and ketoconazole may be seen if these medications are co-administered with PIs and interactions may be seen with certain oral contraceptives.

Many PIs are combined with low dose ritonavir in adults (boosted PI or pharmacoenhancer). Ritonavir inhibition of the cytochrome p450 3A4 enzyme results in diminished metabolism of the second PI. However, many boosted PI combinations have not been studied in HIV-infected children, and there is potential for enhanced drug interactions and hyperlipidemia. Current guidelines recommend the use of three boosted PI regimens (lopinavir, atazanavir and fosamprenavir) in HIV-infected children. ${ }^{3}$

\section{Lopinavir/ritonavir (Kaletra ${ }^{\circledR}$ )}

This co-formulated product is the preferred PI in children. It is available both as a liquid and a tablet, and may be administered to infants as young as two weeks. ${ }^{3}$ Although co-administration with food is required for the liquid formulation, the tablet may be administered with or without food. The liquid formulation has a bitter taste, resulting in a poor patient acceptance rate. Common side effects include gastrointestinal symptoms such as nausea, vomiting, and diarrhea, rash, asthenia, lipid abnormalities, and headache. Changes in body fat composition occur less commonly than with other PIs, but lopinavir/ritonavir does interact with multiple medications including atorvastatin, ethinylestradiol, rifabutin, ketoconazole, itraconazole, and calciumchannel blockers. ${ }^{33}$ Increased dosages of lopinavir/ritonavir are required if this medication is co-administered with nevirapine or efavirenz. Lopinavir/ritonavir has proven virologic and immunologic efficacy in the treatment of both treatment-naïve and treatment-experienced HIV-infected children., ${ }^{9,33-35}$

\section{Nelfinavir $\left(\right.$ Viracept $\left.^{\circledR}\right)$}

Nelfinavir was the first PI recommended for pediatric use and has been extensively studied in children. ${ }^{9,36}$ At the 
present time, nelfinavir is recommended as an alternative PI for children aged $\geq 2$ years. ${ }^{3}$ The liquid formulation is compounded by mixing nelfinavir powder with water. Nelfinavir requires administration with food and is usually dosed twice daily, but younger children require thrice daily administration. Nelfinavir is well-tolerated; diarrhea is the most common side effect. Other frequent side effects include asthenia, abdominal pain, rash, and lipid abnormalities. Less commonly, fat redistribution and exacerbation of chronic liver disease may occur. ${ }^{30}$ In 2007, nelfinavir was temporarily banned for use in children aged $\leq 2$ years after it was found to contain ethyl methanesulfonate (EMS), a potential carcinogen. ${ }^{37}$ However, the product was reformulated and now meets acceptable EMS limits as per the FDA recommendation for use in children and pregnant women. ${ }^{3,38}$ A number of drug interactions are seen between nelfinavir and certain antibiotics, proton pump inhibitors, corticosteroid preparations, and benzodiazepines. In addition, nelfinavir may interact with other ARV medications including a number of protease inhibitors, didanosine, and nevirapine.

\section{Atazanavir $\left(\right.$ Reyataz $\left.^{\circledR}\right)$}

Boosted atazanavir is currently recommended as an alternative PI in children $\geq 6$ years. Use of unboosted atazanavir in children aged $\leq 13 \mathrm{yrs}$ (and/or $\leq 39 \mathrm{~kg}$ ) is generally not recommended due to lack of pediatric dosing information, but may be considered for pediatric use under special circumstances (children aged $\geq 13$ years weighing $\geq 39 \mathrm{~kg}$ ). ${ }^{3,39}$ Atazanavir is administered once daily with food. Its most common side effect is asymptomatic hyperbilirubinemia but jaundice, headache, fever, arthralgia, depression, insomnia, dizziness, nausea, vomiting, diarrhea, and paresthesias may occur. PR interval prolongation has been reported with first-degree and second degree atrioventricular block. Therefore atazanavir should be used cautiously in patients with arrhythmias, or those receiving other agents such as calcium channel blockers that may affect the PR interval. Skin rash is most often mild to moderate, but Stevens-Johnson syndrome has been reported. Compared with other PIs, atazanavir has less effect on serum lipids and causes less fat redistribution. Use of atazanavir is also complicated by numerous drug interactions, as it is both a substrate and inducer of the CYPA4 enzyme system. Only boosted atazavanir should be co-administered with tenofovir. As efavirenz and nevirapine may decrease plasma levels of atazanavir, nevirapine co-administration should be avoided; efavirenz and boosted atazanavir may be co-administered only in treatment-naïve patients but not in treatment-experienced patients. Caution is also needed with co-administration of $\mathrm{H} 2$-receptor antagonists and proton pump inhibitors. Atazanavir should be given one hour after buffered didanosine, if these 2 medications are given together. ${ }^{39}$

\section{Fosamprenavir (Lexiva ${ }^{\circledR}$ )}

Fosamprenavir is a prodrug of amprenavir that is formulated as both a liquid and a tablet that may be administered with or without food. Boosted fosamprenavir is currently recommended as an alternative PI in children aged $\geq 6$ years, and for use without ritonavir in children aged $\geq 2$ years under special circumstances. It is administered once daily to adults but twice daily dosing is recommended for HIV-infected children. ${ }^{3}$ Selection of dosing regimen depends upon whether the patient is treatment-naïve or treatment-experienced. The main advantage of fosamprenavir use is a low pill burden; numerous drug interactions are the major drawback to its use. Side effects include rash, gastrointestinal symptoms, headache, perioral paresthesias, and lipid abnormalities. Less common side effects include severe rash, fat redistribution, neutropenia, and elevation of serum creatinine levels. Fosamprenavir is a sulfonamide that should be used cautiously in patients reporting sulfonamide allergy. ${ }^{39}$

\section{Ritonavir (Norvir ${ }^{\circledR}$ )}

Ritonavir has been available for long-term pediatric use. This potent PI is available as a liquid formulation that must be administered with food, but has a poor acceptance rate due to its bitter taste. Numerous drug interactions due to inhibition of the cytochrome P450 isoenzyme CYP3A complicate its use. ${ }^{30}$ Side effects include gastrointestinal symptoms, headaches, circumoral paresthesias, and lipid abnormalities. Less commonly, fat redistribution and exacerbation of chronic liver disease may occur. Caution is recommended when administering this drug to patients with moderate-severe hepatic impairment. PR interval prolongation and allergic reactions including urticaria, angioedema, and bronchospasm have also been reported. ${ }^{9}$

\section{Saquinavir (Invirase ${ }^{\circledR}$ )}

Saquinavir is not approved for use in children but is recommended for use in combination with low dose ritonavir under special circumstances. ${ }^{3}$ Currently, it is available only in a soft gel tablet form that is administered twice daily with food. Side effects include gastrointestinal intolerance, paresthesias, headache, rash, and lipid abnormalities. Less commonly, fat redistribution and exacerbation of chronic liver disease may occur. Due to low bioavailability, this PI should never be used as a 
sole PI and requires boosting with another PI such as ritonavir. ${ }^{30}$ Numerous drug interactions also complicate its use.

\section{Indinavir (Crixivan ${ }^{\circledR}$ )}

Indinavir is a PI administered only via capsule that requires thrice daily dosing. It is not available in liquid formulation and the risk of renal toxicity (hematuria, nephrolithiasis) limits its use in children. However, it may be considered for use, along with ritonavir boosting, in adolescents who weigh enough to receive adult dosing. It should be taken on an empty stomach and patients receiving this PI should be advised to increase their fluid intake. ${ }^{30}$

Two other PIs commonly used for the treatment of HIVinfected adults, but not recommended for use in children at this time include tipranavir and darunavir. Tipranavir (Aptivus $^{\circledR}$ ) has proven useful for PI-experienced adults with multiple PI mutations in the viral genome. Although it was approved for use in children aged 2 through 18 years in 2008 , lack of pediatric data limits its use in children. It is not recommended for initial therapy but may be useful in children with treatment failure. ${ }^{3}$ It is available as a tablet and an oral solution and may be given with or without food. Drug interactions occur frequently. Common side effects include gastrointestinal symptoms, fatigue, headache, rash, lipid abnormalities, and serum transminase elevation. Less common side effects include fat redistribution, hepatitis/hepatic decompensation, and epistaxis. Rarely, intracranial hemorrhage has been seen. Darunavir (Prezista ${ }^{\circledR}$ ), a potent PI with activity against multidrug-resistant HIV, was approved for use in HIV-infected adults in 2006. Although it was approved for children aged $\geq 6$ years in 2008 , this medication is not recommended for initial therapy in children secondary to high pill burden and limited pediatric data. However, its use may be considered in children with treatment failure. ${ }^{3}$ It is available only in a tablet that should be given with food, and boosted with ritonavir. Side effects include gastrointestinal symptoms (nausea, vomiting, diarrhea, abdominal pain), headache, and fatigue. Less commonly, rash, fever, lipid abnormalities, and serum transminase elevation have been reported. Numerous drug interactions have also been described. Both darunavir and tipranavir contain a sulfonamide component and should be used cautiously in patients reporting sulfonamide allergy. Amprenavir $\left(\right.$ Agenerase $^{\circledR}$ ) is a PI that is no longer manufactured and has largely been replaced by fosamprenavir.

\section{Fusion inhibitors}

Fusion inhibitors inhibit the entry of HIV-1 into host cells by preventing fusion of the viral and cell membranes.

\section{Enfuvirtide $\left(\right.$ Fuzeon $^{\circledR}$ )}

Enfuvirtide is a synthetic 36 -amino acid peptide that binds to the gp41 of the HIV viral envelope. It is administered twice daily via subcutaneous injection. ${ }^{40}$ Enfuvirtide is recommended for use in treatment-experienced HIV-positive adults with advanced HIV infection. Although it was approved for children aged $\geq 6$ years, its use is recommended only in cases of treatment failure. Most patients experience injection site reactions, which are usually mild. ${ }^{41}$ Increased rates of bacterial pneumonia and local site cellulitis have been reported. Rare systemic hypersensitivity reactions have been reported in $<1 \%$ of patients and require permanent discontinuation of this medication.

\section{Entry inhibitors}

Entry inhibitors block the binding of HIV-1 to the human CCR5 chemokine receptor, preventing viral entry into the CD4 cell.

\section{Maraviroc (Selzentry ${ }^{\circledR}$ )}

Maraviroc is an R5-specific inhibitor approved in 2007 for use in HIV-infected patients aged $\geq 16$ years with $R 5$ - tropic virus and multidrug-resistant virus. A co-receptor tropism assay should be performed prior to the initiation of therapy with any CCR5 inhibitor to confirm that a patient's dominant virus population is $\mathrm{R}-5$, rather than X4. Only patients with R5- tropic virus would be expected to respond to Maraviroc; it is ineffective against those with dual-tropic or predominantly X-4 virus. Maraviroc is not approved for use in children aged $\leq 16$ years, but may be useful in cases of treatment failure. Common side effects include cough, fever, upper respiratory infection (URI), rash, musculoskeletal symptoms, abdominal pain, and dizziness. Less common side effects include cardiovascular abnormalities and hepatic failure. There are multiple drug interactions which may affect its dosing. It may be given with or without food, but decreased absorption occurs with fatty meal administration. ${ }^{6,42}$

\section{Integrase inhibitors}

Integrase inhibitors inhibit proviral DNA-strand transfer, interfering with insertion of viral DNA into the host genome.

\section{Raltegravir (Isentress ${ }^{\circledR}$ )}

Raltegravir was approved in 2007 for use in treatmentexperienced HIV-infected patients aged $\geq 16$ years with multidrug-resistant virus. It is dosed twice daily in adults but lack of pediatric data and unavailability of a pediatric formulation limit its use in children at this time. ${ }^{43,44}$ Side effects include diarrhea, nausea, URI symptoms, and dizziness. 


\section{Fixed drug combinations}

A number of fixed drug combination (FDCs) have proven convenient for ART administration in adults. There are currently five FDCs available including Combivir ${ }^{\circledR}$, Trizivir $^{\circledR}$, Epzicom $^{\circledR}$, Truvada $^{\circledR}$, and Atripla ${ }^{\circledR}$. Combivir ${ }^{\circledR}$ consists of zidovudine/lamivudine; Trizivir ${ }^{\circledR}$ contains zidovudine, lamivudine, and abacavir. Lamivudine and abacavir are combined within Epzicom ${ }^{\circledR}$ while Truvada ${ }^{\circledR}$ contains combined emtricitabine and tenofovir. Atripla ${ }^{\circledR}$ is a once daily pill containing emtricitabine, tenofovir, and efavirenz. The main advantage of FDCs is ease of use with lower pill burdens. However, providing the correct dose for children may be difficult with FDCs, and underdosage may occur in children who may require higher doses relative to body weight compared with adults. ${ }^{37}$

\section{Future treatment/novel therapies}

Pediatric studies are currently under way to determine the safety and efficacy of ARV agents such as etravirine and tenofovir, which are currently approved for HIV-infected adults, but not for children. ${ }^{20}$ NRTIs in development for adult use include elvicitabine, racivir and apricitabine, while rilpivirine is a second generation NNRTI under study ${ }^{45}$ Sifuvirtide is a next-generation fusion inhibitor in development. ${ }^{46}$ Experimental chemokine receptor inhibitors include the CCR5 antagonist Vicriviroc and CXCR4 antagonists. ${ }^{47,48}$ Elvitegravir is a once daily integrase inhibitor being studied in adults. Ibalizumab is an anti-CD4 monoclonal antibody that interferes with HIV viral entry. New classes of ART under investigation include inhibitors of viral transcription, translation, and maturation.

\section{Recommendations from current pediatric guidelines}

The first guidelines for treatment of pediatric HIV infection were issued by the US Working Group on Antiretroviral Therapy and the Medical Management of HIV-Infected Children in 1993 and have since undergone numerous revisions. These guidelines include recommendations for the selection of initial and subsequent regimens, and contain both preferred and alternate regimens for the treatment of HIV-infected children, as well as detailed prescribing information for all pediatric and adolescent ARV medications. ${ }^{3}$

\section{Initiation of antiretroviral therapy in antiretroviral-naïve children}

The decision to initiate ART is based upon disease severity, risk of disease progession, and availability of appropriate and palatable drug formulations (Table 2). Benefits of early therapy include preservation of immune function, prevention of disease progression, and possible lowering of the viral set point. Delaying the initiation of therapy may promote improved future medication adherence, reduce drug toxicity, and minimize drug resistance. Although the optimal timing of initial therapy is controversial in HIV-infected adults, most HIV specialists agree that ART is indicated for all HIV-infected infants aged $<1$ year as they are at high risk of disease progression. Early ART has been shown to decrease the risk of AIDS and death in perinatally-infected infants. ${ }^{49}$ Treatment is also recommended for any child aged $\geq 1$ year with moderate (most clinical category B conditions) or severe symptoms, including AIDS (Category C), regardless of CD4 count or HIV RNA level as HIV disease progression is likely to occur in these children (Table 3). ${ }^{50}$ Current guidelines also advocate treatment of any child aged $>1$ year with CD4 lymphocytopenia for age, and for older children ( $\geq 5$ years) who are symptomatic or have CD4 lymphocytopenia for age (Table 2). Thus, ART would be indicated for children aged 1 to 5 with CD4 $<25 \%$, or children aged $\geq 5$ years with CD $<350$ cells $/ \mathrm{mm}^{3}$.

ART may be considered for children aged $\geq 1$ year with normal CD4 for age and high viral load (plasma RNA $\geq 100,000$ copies $/ \mathrm{mL}$ ), even in the absence of symptoms or presence of mild symptoms (clinical categories $\mathrm{N}$, A, or the selected clinical category $\mathrm{B}$ conditions of bacterial infection or lymphoid interstitial pneumonitis (LIP). Treatment may be deferred in children aged $\geq 1$ year who lack symptoms or have mild symptoms, and have normal CD4 for age and HIV RNA $<100,000$ copies/mL, as they are at low risk for HIV disease progression. Untreated children should be monitored closely over time with periodic CD4 counts, HIV RNA levels, and clinical follow-up to determine whether treatment should be initiated.

Treatment readiness and the ability of the child and the caregiver to adhere to a prescribed regimen should be assessed prior to the initiation of therapy. Comorbid conditions such as tuberculosis, hepatitis B or C infection, or renal or liver disease may influence choice of ARV medication. Laboratory assessment prior to initiation of therapy should include CD4 count/percentage, HIV RNA level, viral resistance testing (genotype or phenotype), complete blood count with differential, serum chemistries including hepatic enzymes, serum lipase and amylase, and serum lipids (triglycerides and cholesterol). CD4 percentage is preferred for immunologic monitoring of children aged $\leq 5$ years, whereas absolute CD 4 count is a better immunologic parameter in older children.${ }^{51}$ Five viral load 
Table 2 Indications for treatment of HIV-infected children. Adapted from the Guidelines for the Use of Antiretroviral Agents in Pediatric HIV Infection ${ }^{3}$

\section{INITIATE TREATMENT}

All infants $<$ I year

Any infant or child with AIDS or significant HIV symptoms (Clinical Category B*,C)

Child ages $\mathrm{I}-5$ years with CD4 $\leq 25 \%$ (regardless of symptoms or HIV RNA level)

Child $\geq 5$ years with CD4 $\leq 350$ cells $/ \mathrm{mm}^{3}$

\section{CONSIDER TREATMENT}

Child I-5 years with CD4 $\geq 25 \%$ and HIV RNA $\geq 100,000$ copies $/ \mathrm{mL}$ (regardless of symptoms)

Child $\geq 5$ years with CD4 $\geq 350$ cells $/ \mathrm{mm}^{3}$ and HIV RNA $\geq 100,000$ copies $/ \mathrm{mL}$

\section{DEFER TREATMENT}

Child age I-5 years with no symptoms/mild symptoms** + CD4 $\geq 25 \%+$ HIV RNA $\leq 100,000$ copies/mL

Child age $>5$ years with no symptoms/mild symptoms** + CD4 $>350$ cells $/ \mathrm{mm}^{3}$ and HIV RNA $<100,000 \mathrm{copies} / \mathrm{mL}$

Notes: *except single episode of bacterial infection or LIP. ***with a single episode of bacterial infection or LIP.

assays have been approved by the FDA for quantitation of HIV RNA copy number. None is preferred but continued use of the same assay over time is recommended for individual patients.

\section{Types of regimens}

Most regimens include triple drug combinations consisting of two NRTIs with either an NNRTI (NNRTI-based regimen) or a boosted PI (PI-based regimen) (Table 4). Under special circumstances, a triple NRTI regimen consisting of zidovudine, lamivudine, and abacavir may be used. ${ }^{3}$ Advantages of NNRTI-based regimens include ease of administration with low pill burden and decreased incidence of lipid abnormalities and fat maldistribution, compared with PI-based regimens. ${ }^{37}$ However, cutaneous side effects as well as potential drug interactions, and development of cross-resistance among NNRTIs are drawbacks to these regimens.

PI-based regimens are potent but their use is complicated by high pill burdens, frequent drug interactions, and drug toxicity. ${ }^{37}$ The main advantage of these regimens is a high genetic barrier to the development of drug resistance. ${ }^{9}$ Triple NRTI regimens are generally well-tolerated by children as they have few side effects, pediatric formulation availability, and ease of use. ${ }^{18}$ This type of regimen will preserve NNRTIs and PIs for future use. Also, because of the a risk of a life-threatening hypersensitivity reaction to abacavir, use of the triple NRTI regimen containing zidovudine, lamivudine, and abacavir is recommended only under special circumstances when a preferred or alternate NNRTI-based or PI-based regimen cannot be used. ${ }^{3}$ Other
NRTI combinations such as tenofovir/abacavir/lamivudine or tenofovir/didanosine/lamivudine are not recommended as initial therapy for HIV-infected children due to inferior virologic potency. ${ }^{52}$

The "Guidelines for the Use of Antiretroviral Agents in Pediatric HIV Infection"contain both preferred and alternate dual NRTI combinations to be used with either an NNRTI or PI-based regimens (Table 4). These various NRTI combinations have been studied and proven safe, effective, and durable in children. ${ }^{13,16,36,53,54}$ The combination of zidovudine and lamivudine is the most studied in children and is well-tolerated. ${ }^{11}$ Although less data is available on its pediatric use, the similarity of emtricitabine led to its selection as a possible substitute for lamivudine for dual NRTI use. ${ }^{16}$ The therapeutic efficacy of zidovudine in HIV-infected children was first reported by the AIDS Clinical Trials Group (ACTG) 152 study team, which compared zidovudine monotherapy, didanosine monotherapy, and combined zidovudine/didanosine in $831 \mathrm{HIV+}$ symptomatic children. Combined zidovudine/didanosine therapy and didanosine monotherapy were proven superior to zidovudine.$^{55}$ A 2001 study compared the safety, tolerability, and efficacy of dual zidovudine/lamivudine versus triple therapy with zidovudine/lamivudine/abacavir in a cohort of 205 children and reported superiority of the three-drug combination compared with the two-drug regimen. ${ }^{18}$

The "Guidelines for the Use of Antiretroviral Agents in Pediatric HIV Infection" also lists ARV medications that are not recommended for use in HIV-infected children at any time. Single agent monotherapy or dual NRTI therapy 
Table 31994 Revised human immunodeficiency virus pediatric classification system; clinical categories (updated February 28, 2008) (50 $^{5}$

Category N: Not symptomatic: Children who have no signs of symptoms considered to be the result of HIV infection or who have only one of the conditions listed in Category A.

Category A: Mildly symptomatic: Children with two or more of the following conditions listed in categories B and C:

Lymphadenopathy ( $\geq 0.5 \mathrm{~cm}$ at more than two sites: bilateral at one site)

Hepatomegaly

Splenomegaly

Dermatitis

Parotitis

Recurrent or persistent upper respiratory infection, sinusitis, or otitis media

Category B: Moderately symptomatic: Children who have symptomatic conditions, other than those listed for category A or category C, that are attributed to HIV infection. Examples of conditions in clinical category B include, but are not limited to, the following:

Anemia $(<8 \mathrm{gm} / \mathrm{dL})$, neutropenia $\left(<1000\right.$ cells $\left./ \mathrm{mm}^{3}\right)$, or thrombocytopenia $\left(<100,000\right.$ cells $\left./ \mathrm{mm}^{3}\right)$ persisting $\geq 30$ days

Bacterial meningitis, pneumonia, or sepsis (single episode)

Candidiasis, oropharyngeal (ie,thrush) persisting for $>2$ months in children aged $>6$ months

Cardiomyopathy

Cytomegalovirus infection with onset before age I month

Diarrhea, recurrent or chronic

Hepatitis

Herpes simplex virus (HSV) stomatitis, recurrent (ie, more than two episodes within I year)

HSV bronchitis, pneumonitis, or esophagitis with onset before age I month

Herpes zoster (ie, shingles) involving at least two distinct episodes or more than I dermatome

Leiomyosarcoma

Lymphoid interstitial pneumonitis (LIP) or pulmonary lymphoid hyperplasia complex

Nephropathy

Nocardiosis

Fever lasting $>$ I month

Toxoplasmosis with onset before age I month

Varicella, disseminated (ie, complicated chickenpox)

Category C: Severely symptomatic: Children who have any condition listed in the 1987 surveillance case definition for acquired immunodeficiency syndrome (below) with the exception of LIP (which is a category B condition)

Serious bacterial infections, multiple or recurrent (ie, any combination of at least two culture-confirmed infections within a 2 year period) of the following types: septicemia, pneumonia, meningitis, bone or joint infection, or abcess of an internal organ or body cavity (excluding otitis media, superficial skin or mucosal abcesses, and indwelling catheter-related infections)

Candidiasis, esophageal or pulmonary (bronchi, trachea, lungs)

Coccidioidomycosis, disseminated (at site other than or in addition to lungs or cervical or hilar lymph nodes)

Cryptococcosis, extrapulmonary

Cryptosporidiosis or isosporiasis with diarrhea persisting $>I$ month

Cytomegalovirus disease with onset of symptoms at age $>$ I month (at a site other than liver, spleen, or lymph nodes)

Encephalopathy (at least I of the following progressive findings present for at least two months in the absence of a concurrent illness other than HIV infection that could explain the findings): a) failure to attain or loss of developmental milestones or loss of intellectual ability, verified by standard developmental scale or neuropsychological tests; b) impaired brain growth or acquired microcephaly demonstrated by head circumference measurements or brain atrophy demonstrated by computerized tomography or magnetic resonance imaging (serial imaging required for children $<2$ years of age); $c$ ) acquired symmetric motor deficit manifested by 2 or more of the following: paresis, pathologic reflexes, ataxia, or gait disturbance

Herpes simplex virus infection causing a mucocutaneous ulcer that persists for $>I$ month; or bronchitis, pneumonitis, or esophagitis for any duration affecting a child $>$ I month of age

Histoplasmosis, disseminated (at a site other than or in addition to lungs or cervical or hilar lymph nodes)

Kaposi's sarcoma

Lymphoma, primary in brain

Lymphoma, small, noncleaved (Burkitt's), or immunoblastic or large cell lymphoma of B-cell or unknown immunologic phenotype

(Continued) 
Table 3 (Continued)

\begin{tabular}{l} 
Mycobacterium tuberculosis, disseminated or extrapulmonary \\
Mycobacterium, other species or unidentified species, disseminated (at site other than or in addition to lungs, skin, or cervical or hilar lymph nodes) \\
Mycobacterium avium complex or Mycobacterium kansasii, disseminated (at site other than or in addition to lungs, skin, or cervical or hilar lymph \\
nodes) \\
Pneumocystis jiroveci pneumonia \\
Progressive multifocal leukencephalopathy \\
Salmonella (nontyphoid) septicemia, recurrent \\
Toxoplasmosis of the brain with onset at $>$ I month of age \\
Wasting syndrome in the absence of a concurrent illness other than HIV infection that could explain the following findings: a) persistent weight loss $>$ I0\% \\
of baseline; OR b) downward crossing of atleast two of the following percentile lines on the weight-for-age chart (eg, 95 th, 75 th, 50 th, 25 th, 5 th) in a child $\geq I$ \\
year of age; OR c) $<5$ th percentile on weight-for-height chart on two consecutive measurements, $\geq 30$ days apart PLUS I) chronic diarrhea (ie, $>2$ loose \\
stools per day for $\geq 30$ days), OR 2 ) documented fever for $\geq 30$ days, intermittent or constant) \\
\hline
\end{tabular}

should not be given, except for the use of zidovudine monotherapy in prenatal HIV exposure prophylaxis. Certain NRTI combinations (zidovudine/stavudine, lamivudine/emtricitabine, didanosine/stavudine) are not recommended for use as part of a HAART regimen. Other ARV medications not recommended for pediatric use include tenofovir, efavirenz (in the first trimester of pregnancy or in sexually active females of childbearing potential), and nevirapine (in adolescent females with CD4 $>250$ cells $/ \mathrm{mm}^{3}$ or adolescent males with CD4 $>400$, unless the benefits of therapy outweigh the risks). Pediatric use of unboosted saquinavir or combined indinavir/atazanavir is also not recommended. ${ }^{3}$

In addition, the "Guidelines for the Use of Antiretroviral Agents in Pediatric HIV Infection" include ARV medications/ART regimens that lack sufficient pediatric data at this time for use as initial therapy in treatment-naïve children. However, use of these agents may be considered for secondary therapy in certain treatment-experienced or older children/adolescents. ${ }^{3}$ Use of dual PI regimens or use of boosted PI regimens other than the three recommended alternate regimens (lopinavir/ritonavir, atazanavir/ritonavir, and fosamprenavir/ritonavir) are not recommended nor is use of unboosted atazanavir in children aged $\leq 13$ years or $\leq 39 \mathrm{~kg}$. Three-class regimens (NRTI + NNRTI + PI) should not be used. Contraindicated NRTI combinations include zidovudine/stavudine, lamivudine/emtricitabine, and stavudine/didanosine. Use of agents in newer classes including tipranavir, darunavir, maraviroc, raltegravir, etravirine, and enfuvirtide is also not recommended for initial therapy in HIV-infected children or adolescents at the present time but may be useful in cases of treatment failure. Indinavir has studied mostly in small uncontrolled trials in HIV-infected children, and is not currently FDA-approved in the pediatric age group, nor is it recommended for use as initial therapy.

\section{Monitoring of children receiving antiretroviral therapy}

HIV-infected children and adolescents should be closely monitored following the initiation of ART to determine whether the prescribed regimen is tolerable, and whether side effects are occurring. This is especially important during the first few weeks of therapy when adherence may be compromised. Therefore, clinical or telephone follow-up one to two weeks after starting new medication or undergoing a regimen change is recommended. Treated children should return to the clinic one month after beginning a new regimen for clinical and laboratory assessment consisting of complete blood count and differential, serum chemistries, serum lipids, CD4 count/ percentage, and HIV RNA level. Increases in CD4 cell count/percentage and decreases in HIV RNA levels signify a response to ART. Ideally, ART should reduce HIV RNA levels to undetectable levels (HIV RNA $<50$ copies $/ \mathrm{mL}$ ). ART-treated patients should then be seen at regular intervals (every 3-4 months) to ascertain proper medication adherence, monitor for drug side effects, and to ensure efficacy of the medical regimen. CD4 count/percentage and plasma HIV RNA should be checked every 3-4 months. Serum amylase and lipase should be monitored in patient receiving didanosine and stavudine. A lipid panel should be obtained every $6-12$ months. ${ }^{3}$

Immune reconstitution inflammatory syndrome (IRIS) may be seen in children during the first three months of initiating a new ART regimen. This occurs clinically as a worsening of symptoms of inflammation or infection due to increases in CD4 count. At this time, treatment of IRIS 
is largely empiric, although antibiotics, antivirals, and corticosteroids have been used. ${ }^{56}$

\section{Management of the treatment- experienced child/treatment failure}

A prompt change in the ART regimen should be considered for HIV-infected patients who are experiencing clinical HIV disease progression, immunologic deterioration, drug resistance, or increasing HIV viral replication. Clinical features that may warrant a change in regimen include progressive neurodevelopmental deterioration, growth failure, or recurrent or severe infections. Any decreases in CD4 count or increases in HIV RNA levels should be confirmed by repeat laboratory testing prior to a medication switch. CD4 levels are based on age and absolute counts and percentages, and will normally decrease over time in children. Successful ART therapy should result in a $>1.0 \log _{10}$ decline in HIV RNA level from baseline after two to three months of therapy, but a slower decline may be seen in patients with higher initial HIV RNA levels. HIV RNA levels $>400$ copies $/ \mathrm{mL}$ after six months of treatment or detectable HIV RNA after one year of treatment may represent virologic failure. It has been estimated that $30 \%-80 \%$ of HIV-infected children will experience treatment failure within one year of treatment initiation. ${ }^{14}$ Children with treatment failure should be evaluated for medication adherence, drug intolerance, and possible drug interactions which may lessen the efficacy of the therapeutic regimen. Frequent medication regimen changes are not advisable for HIV-infected children since this may limit future treatment options. Thus, in certain circumstances, children may benefit from continuation of an ART regimen with more frequent monitoring of CD4 counts and HIV RNA levels.

Careful review of ART medication history and past drug resistance testing is recommended prior to initiating any medication change, as is a complete history and physical examination. When selecting a new regimen, it is important

Table 4 Initial therapy for HIV-infected children. Adapted from the Guidelines for the Use of Antiretroviral Agents in Pediatric HIV Infection $^{3}$

\begin{tabular}{ll}
\hline $\begin{array}{l}\text { A. NNRTI-BASED REGIMEN: } \\
\text { Preferred regimen }\end{array}$ & Alternative regimen \\
Efavirenz +2 NRTIs $(\geq 3$ years) & Nevirapine +2 NRTIs ( $\geq 3$ years) \\
Nevirapine +2 NRTIs $(<3$ years) & (child unable to swallow capsule) \\
$\begin{array}{l}\text { B. PI-BASED REGIMEN } \\
\text { Preferred regimen }\end{array}$ & \\
Lopinavir/ritonavir +2 NRTIs & Alternative regimen \\
& Atazanavir/ritonavir + 2 NRTls \\
(children $\geq 6$ years)
\end{tabular}

Special circumstances

Fosamprenavir (unboosted) +2 NRTls

(children $\geq 2$ years)

Atazanavir (unboosted) +2 NRTIs

(for treatment-naïve

adolescents $>3$ years and $>39 \mathrm{~kg}$ )

Zidovudine + Lamivudine + Abacavir

Dual NRTI backbones for use in above regimens (A and B)

Preferred regimen
Lamivudine + Abacavir*
Emtricitabine + Abacavir*
Didanosine + Emtricitabine
Zidovudine + Lamivudine
Zidovudine + Emtricitabine
Tenofovir** + Lamivudine
Tenofovir** + Emtricitabine

Notes: *HLA B*570I genetic testing recommended prior to therapy with use of abacavir contraindicated in patients found to be HLA B*570I-positive. **For Tanner Stage 4 or post-pubertal adolescents only who are unable to tolerate ritonavir + 2 NRTls (boost with ritonavir if tenofovir used). Saquinavir/ ritonavir +2 NRTIs for post-pubertal adolescents who weigh enough to receive adult doses).

\section{Alternative regimen}

Zidovudine + Abacavir*

Zidovudine + Didanosine

\section{Special circumstances}

Stavudine + Lamivudine

Stavudine + Emtricitabine 
to discontinue all medications to which the patient's virus is resistant, and to avoid the initiation of any agents with possible cross resistance, to ensure that all agents are fully active. Cross resistance occurs commonly among NRTIs and NNRTIs. Another consideration in changing regimens is the preservation of future treatment options including use of novel agents. Caregivers should always discuss adherence issues and select a medication regimen that is acceptable to the patient in terms of drug formulation, pill burden, dosing frequency, and meal requirements, prior to prescribing a new antiretroviral regimen.

For children failing their first PI-based regimen, a change to an NNRTI-based regimen is recommended. Conversely, children failing a NRTI-based regimen should switch to a PI-based regimen. At least two and preferably three active agents should be used. In certain situations, use of a triple class regimen may be necessary if a potent dual NRTI backbone cannot be identified. Consultation with a pediatric HIV specialist is recommended in patients with multidrug-resistant virus or those with limited treatment options. In the case of treatment-experienced children aged $\geq 16$ years failing their ART regimen, use of certain newer medications such as darunavir, maraviroc, or raltegravir may be considered. Enfuvirtide may also be useful for use in heavily treatment- experienced children and adolescents. ${ }^{3}$

\section{Drug resistance}

Viral resistance testing is recommended prior to initiation of ART or modification of a failing treatment regimen. Failure to maximally suppress viral replication may result in viral mutations that lead to drug resistance. However, a lack of drug resistance does not ensure that a medication will successfully reduce viral replication. Resistance assays may be genotypic (GT) or phenotypic (PT). GT assays detect specific viral mutations in patients with HIV RNA levels $>1,000$ copies/mL. PT assays directly assess whether a viral isolate can grow in the presence of an ARV medication, measuring the $50 \%$ or $90 \%$ minimal inhibitory concentrations of a drug against the virus in vitro compared with a laboratory strain of wild-type virus. A third type of resistance assay (virtual phenotype) predicts the phenotype based on viral genotype. Although a number of resistance assays are commercially available, none is preferred for use in adults or children. However, continued use of the same type of resistance assay over time is recommended for individual patients. The assay should be performed before or within four weeks of drug discontinuation since reversion to wild-type virus may occur within four to six weeks of regimen discontinuation. Careful review of antiretroviral medication history and consultation with a pediatric HIV specialist may be needed for interpretation of viral resistance data. The International AIDS Society-USA (IAS-USA) maintains a list of mutations associated with clinical resistance to HIV which is updated regularly. ${ }^{57}$ Medication nonadherence should be suspected if persistent viremia is seen without evidence of viral resistance.

\section{Medication adherence}

Patients with poor medication adherence are at risk for the development of mutations and viral resistance. There are unique medication adherence issues that affect specific age groups. The caregiver/child relationship, HIV disclosure issues, and unpalatable drug formulations may adversely impact proper medication adherence in HIV-infected children, while denial of illness, lack of social support, and mental illness may impede proper ART administration in HIV-positive adolescents. For all treated patients, medication adherence must be evaluated at all medical visits. Any barriers to adherence should be promptly identified and addressed. Some patients may benefit from the use of adherence aids such as medication timers, beepers, or diaries, while others may require the use of intensive pharmacologic or nursing services such as special medication packaging, or directly observed therapy. To ensure the success of an ART regimen, providers should simplify the medical regimen, using the lowest pill burden and formulations that are acceptable to the patient. ${ }^{58}$

\section{Discontinuation/Interruption of therapy}

There are certain situations where temporary discontinuation of ART may be indicated. These include significant drug toxicity, acute gastrointestinal illness, surgery, sedation, or patient/caregiver request. Severe medication toxicity necessitates complete discontinuation of all ARV medications, but children with mild or moderate drug toxicity may not require an immediate change to the regimen, as some symptoms may resolve over time or be managed expectantly. ARV dose reduction is generally not recommended except in the case that therapeutic drug monitoring is available. ${ }^{3}$ Structured treatment interruptions (STIs) have been studied in HIV-infected adults to reduce drug toxicity, medication costs, and to provide virologic modification, that is, to return the patient's virus to the wild-type virus state. ${ }^{31}$ However, overall immunological results with STIs have been disappointing, and since minimal data exist regarding STIs in children, STI cannot be recommended at this time. 


\section{Conclusion}

Currently, HIV infection is often a chronic and manageable infection in adults and children. ARV medications from six classes are used sequentially in combination to suppress viral replication to maximal levels. Although the prognosis of HIV infection has improved significantly since the 1980's, viral resistance, drug toxicity, and medication nonadherence still present great challenges to successful treatment. Current practice guidelines for pediatric HIV infection provide updated recommendations to optimize the treatment of HIV infection. ${ }^{3}$ The current complexity and rapidly evolving issues in HIV infection make it highly desirable for providers to consult with pediatric HIV specialists when caring for children infected with HIV. Additional pediatric studies are needed to develop new ARV medications, determine optimal ARV doses for HIV-infected children, enhance medication adherence, and to more effectively assess patients for drug toxicities and potential drug interactions.

\section{Disclosure}

The authors report no conflicts of interest in this work.

\section{References}

1. Scarlatti G. Paediatric HIV infection. Lancet. 1996;349:863-868.

2. Vella S, Floridia M. Antiviral Therapy. In: Cohen and Powderly, editors. Infectious Diseases, New York: Mosby; 2004. Chapter 139.

3. Working Group on Antiretroviral Therapy and the Medical Management of HIV-Infected Children. February, 2009. Guidelines for the Use of Antiretroviral Agents in Pediatric HIV Infection. Accessed March 31, 2009. Available from: http://AIDSinfo.nih.gov.contentfiles/ PediatricGuidelines.pdf.

4. Centers for Disease Control and Prevention. Public Health Service Task Force recommendations for the use of antiretroviral drugs in pregnant women infected with HIV-1 for maternal health and for reducing perinatal HIV-1 transmission in the United States. MMWR 2005. Accessed March 31, 2009. Available from: http://AIDSinfo.nih.gov.

5. 2008 USPHS/IDSA Guidelines for the Prevention of Opportunistic Infections in Persons Infected with Human Immunodeficiency Virus. Accessed March 31, 2009. Available from: http://AIDSinfo.nih.gov.

6. Schlecht HP, Schellhorn S. Dezube BJ, Jacobson JM. New approaches in the treatment of HIV/AIDS- Focus on maraviroc and other CCR5 antagonists. Ther Clin Risk Manag. 2008;4:473-485.

7. Patel K, Hernan MA, Williams PL, et al. Long-term effects of highly active antiretroviral therapy on CD4+ cell evolution among children and adolescents infected with HIV: 5 years and counting. Clin Infect Dis. 2008: 46(11):1751-1760.

8. Rouet F, Fassinou P, Inwoley A, et al. Long-term survival and immunovirological response of African HIV-1 infected children to highly active antiretroviral therapy regimens. Aids. 2006;20(18):2315-2319.

9. Rudin C, Burri M, Shen Y, Rode R, Nadal D. Long-term safety and effectiveness of ritonavir, nelfinavir, and lopinavir/ritonavir in antiretroviral-experienced HIV-infected children. Pediatr Infect Dis J. 2008;27(5):431-437.

10. Luzuriaga K, Bryson Y, Krogstad P, et al. Combination treatment with zidovudine, didanosine, and nevirapine in infants with human immunodeficiency virus type 1 infection. $N$ Engl J Med. 1997;336(19):1343-1349.
11. McKinney RE, Johnson GM, Stanley K, et al. A randomized study of combined zidovudine-lamivudine versus didanosine monotherapy in children with symptomatic therapy-naïve HIV-1 infection. The Pediatric AIDS Clinical Trials Group Protocol 300 Study Team. J Pediatr. 1998;133(4):500-508.

12. Van Dyke RB, Wang L, Williams PL, et al. Toxicities associated with dual nucleoside reverse transcriptase inhibitor regimens in HIV-infected children. J Infect Dis. 2008;198(11):1599-1608.

13. Green H, Gibb DM, Walker AS, et al. Lamivudine/abacavir maintains virological superiority over zidovudine/lamivudine and zidovudine/ abacavir beyond 5 years in children. Aids. 2007;21(8):947-955.

14. Saez-Llorens X, Violari A, Ndiweni D, et al. Long-term safety and efficacy results of once-daily emtricitabine-based highly active antiretroviral therapy regimens in human immunodeficiency virus-infected subjects. Pediatrics. 2008;121(4):e827-e835.

15. Stevens RC, Rodman JH, Yong FH, Carey V, Knupp CA, Frenkel LM. Effect of food and pharmacokinetic variability on didanosine systemic exposure in HIV-infected children. Pediatric AIDS Clinical Trials Group Protocol 144 Study Team. AIDS Res Hum Retroviruses. 2000;16(5):415-421.

16. McKinney RE Jr, Rodman J, Hu C, et al. Long-term safety and efficacy of a once-daily regimen of emtricitabine, didanosine, and efavirenz in HIV- infected, therapy-naïve children and adolescents. Pediatric AIDS Clinical Trials Group Protocol P1021. Pediatrics. 2007;120(2): e146-e23.

17. Cross SJ, Rodman FH, Lindsey JC, et al. Abacavir and metabolite pharmacokinetics in HIV-1 infected children and adolescents. $J$ Acquir Immune Defic Syndr. 2009;51(1):54-59.

18. Saez-Llorens X, Nelson RP, Emmanuel P, et al. A randomized, double-blind study of triple nucleoside therapy of abacavir, lamivudine, and zidovudine versus lamivudine and zidovudine in previously treated human immunodeficiency virus type 1-infected children. Pediatrics. 2001;107(1)e4.

19. Mallal S, Phillips E, Carosi G, et al. HLA B*5701 screening for hypersensitivity to abacavir. $N$ Engl J Med. 2008;358:2514-2516.

20. Gafni RI, Hazra R, Reynolds JC, et al. Tenofovir disoproxil fumarate and an optimized background regimen of antiretroviral agents as salvage therapy: Impact on bone mineral density in HIV-infected children. Pediatrics. 2006;118(3):e711-e718.

21. Riordan A, Judd A, Boyd K, et al. Tenofovir use in human immunodeficiency virus-1-infected children in the United Kingdom and Ireland. Pediatr Infect Dis J. 2009;28(3):204-209.

22. King JR, Acosta EP, Yogev R, et al. Steady state pharmacokinetics of lopinavir/ritonavir in combination with efavirenz in human immunodeficiency virus-infected pediatric patients. Pediatr Infect Dis J. 2009;28(2):159-161.

23. Wintergerst U, Hoffman F, Jansson A, et al. Antiviral efficacy, tolerability and pharmacokinetics of efavirenz in an unselected cohort of HIV-infected children. J Antimicrob Chemotherapy. 2008;61(6): $1336-1339$

24. Starr SE, Fletcher CV, Spector SA, et al. Combination therapy waith efavirenz, nelfinavir, and nucleoside reverse-transcriptase inhibitors in children with human immunodeficiency virus infection type 1. Pediatric AIDS Clinical Trials Group 382 Team. N Engl J Med. 1999;341(25):1874-1881.

25. Fraaij PL, Neubert J, Bergshoeff AS, et al. Safety and efficacy of a NRTI-sparing HAART regimen of efavirenz and lopinavir/ritonvir in HIV-1 infected children. Antivir Ther. 2004;9(2):297-299.

26. Funk MB, Notheis G, Schuster T, et al. Effect of first line therapy including efavirenz and two nucleoside reverse transcriptase inhibitors in HIV-infected children. Eur J Med Res. 2005;10(12):503-508.

27. Verweel G, Sharland M, Lyall H, et al. Nevirapine use in HIV-1 infected children. Aids. 2003;17(11):1639-1647.

28. Luzuriaga K, McManus M, Mofenson L, et al. A Trial of three antiretroviral regimens in HIV-1 infected children. $N$ Engl $J$ Med. 2004;350(24):2471-2480.

29. Baylor M, Ayime O, Truffa M, et al. Hepatotoxicity associated with nevirapine use in HIV-infected children. Boston, MA: 12th Conference on Retroviruses and Opportunistic Infections; February 22-25, 2005. Abstract 776. 
30. van Rossum AM, Fraaij PL, de Groot R. Efficacy of highly active antiretroviral therapy in HIV-1 infected children. Lancet Infect Dis. 2002;2(2):93-102.

31. Department of Health and Human Services. Guidelines for the Use of Antiretroviral Agents in HIV-1-Infected Adults and Adolescents. Accessed March 24, 2009. Available from: http://AIDSinfo.nih.gov/ contentfiles/AdultandadolescentGL.pdf.

32. Gortmaker S, Hughes M, Cervia J, et al. Effect of combination therapy including protease inhibitors on mortality among children and adolescents infected with HIV-1. N Engl J Med. 2001;345(21):1522-1528.

33. Larru B, Resino S, Bellon JM, et al. Long-term response to highly active antiretroviral therapy with lopinavir/ritonavir in pre-treated vertically HIV-infected children. J Antimicrob Chemother. 2008:61(1):183-190.

34. Jankelevich S, Mueller BU, Mackall CL, et al. Long-term virologic and immunologic responses in human immunodeficiency virus type 1-infected children treated with indinavir, zidovudine, and lamivudine. J Infect Dis. 2001;183:1116-1120.

35. Saez-Llorens Xn, Violari A, Deetz C, et al. Forty-eight week evaluation of lopinavir/ritonavir, a new protease inhibitor, in human immunodeficiency virus-infected children. Pediatr Infect Dis J. 2003;22(3):216-223.

36. Scherpier HJ, Bekker V, van Leth F, et al. Long-term experience with combination antiretroviral therapy that contains nelfinavir for up to 7 years in a pediatric cohort. Pediatrics. 2006;117(3):e528-e536.

37. Riordan A, Bugembe T. Update on antiretroviral therapy. Arch Dis Child. 2009;94:70-74.

38. Resino S, Larru B, Maria Bellon J, et al. Effects of highly active antiretroviral therapy with nelfinavir in vertically HIV-1 infected children: 3 years of follow-up: long-term responses to nelfinavir. BMC Infect Dis. 2006;11(6):107.

39. Cunningham D, Freedman A, Read S, et al. Safety and antiviral activity of fosamprenavir-containing regimens in HIV-infected 2- to 18-year old pediatric subjects (Interim data, Study APV29005). Los Angeles, CA: 14th Conference on Retroviruses and Opportunistic Infections; February 25-28, 2007. Abstract 718.

40. Lalezari J, Henry K, O'Hearn M, et al; TORO 1 Study Group. Enfuvirtide, an HIV-1 fusion inhibitor, for drug-resistant HIV infection in North and South /America. N Engl J Med. 2003;348:(22):2175-2185.

41. Kilby JM, Eron JJ. Novel therapies based on mechanisms of HIV-1 cell entry. N Engl J Med. 2003;348(22):2228-2238.

42. Gulick RM, Lalezari, J, Goodrich J, et al. Maraviroc for previously treated patients with R5 HIV-1 Infection. $N$ Engl $J$ Med. 2008;359(14)1429-1441.

43. Hicks C, Gulick RM. Raltegravir: The first HIV type 1 integrase inhibitor. Clin Infect Dis. 2009;48(7):931-939.

44. Brichard B, Van der Linden D. Clinical practice treatment of HIV infection in children. Eur J Pediatr. 2009;168(4)387-392.
45. Fulco PP, McNicholl IR. Etravirine and rilpivirine: nonnucleoside reverse transcriptase inhibitors with activity against human immunodeficiency virus type 1 strains resistant to previous nonnucleoside agents. Pharmacotherapy. 2009;29(3):281-294.

46. Pan C, Lu H, Qi Z, Jiang S. Synergistic efficacy of combination of enfuvirtide and sifuvirtide, the first- and next-generation HIV-fusion inhibitors. Aids. 2009;23(5):639-641.

47. Clay PG. Advances in human immunodeficiency virus therapeutics. Ann Pharmacother. 2006;40(4):704-709.

48. Thakkar N, Pirrone V, Passic S, et al. Specific interactions between the viral coreceptor CXCR4 and the biguanide-based compound NB325 mediate inhibition of human immunodeficiency virus type 1 infection. Antimicrob Agents Chemother. 2009;53(2):631-638.

49. Goetghbuer T, Haelterman E, Le Chenadec J, et al. Effect of early antiretroviral therapy on the risk of AIDS/Death in HIV-infected infants. AIDS 2009;23(5):597-604.

50. Centers for Disease Control and Prevention. 1994 revised classification system for human immunodeficiency virus infection in children less than 13 years of age, 1994 (No. RR-12).p. 1-10.

51. HIV Paediatric Prognostic Markers Collaborative Study. Predictive value of absolute CD4 cell count for disease progression in untreated HIV-1 infected children. Aids. 2006;20(9):1289-1294.

52. Gerstoft J, Kirk O, Obel N, et al. Low efficacy and high frequency of adverse events in a randomized trial of the triple nucleoside regimen abacavir, stavudine, and didanosine. Aids. 2003;17(14):2045-2053.

53. Paediatric European Network for Treatment of AIDS (PENTA). Comparison of dual nucleoside-analogue reverse transcriptase inhibitor regimens with and without nelfinavir in children with HIV-1 who have not previously been treated: the PENTA 5 randomised trial. 2002;359(9308):733-740.

54. Kline MW, van Dyke RB, Lindsey J, et al. Combination therapy with stavudine (d4T) plus didanosine (ddI) in children with human immunodeficiency virus infection. The Pediatric AIDS Clinical Trials Group Protocol 327 Team. Pediatrics. 1999;103(5):e62.

55. Englund JA, Baker CJ, Raskino C, et al. Zidovudine, didanosine, or both as the initial treatment for symptomatic HIV-infected children. AIDS Clinical Trials Group (ACTG) Study 152 Team. $N$ Engl J Med. 1997;336(24):1704-1712.

56. Deeks SG. Antiretroviral treatment of HIV infected adults. BMJ. 2006;332:1489-1503.

57. International AIDS Society-USA (IAS-USA). HIV drug resistance mutations. 2009. Accessed on February 10, 2009. Available from: http://iasusa.org/resistance_mutations/index.html.

58. Anderson PL. Pharmacologic perspectives for once-daily antiretroviral therapy. Ann Pharmacother. 2004;38(11):1924-1934.
Therapeutics and Clinical Risk Management

\section{Publish your work in this journal}

Therapeutics and Clinical Risk Management is an international, peerreviewed journal of clinical therapeutics and risk management, focusing on concise rapid reporting of clinical studies in all therapeutic areas, outcomes, safety, and programs for the effective, safe, and sustained use of medicines. This journal is indexed on PubMed Central, CAS,

\section{Dovepress}

EMBase, Scopus and the Elsevier Bibliographic databases. The manuscript management system is completely online and includes a very quick and fair peer-review system, which is all easy to use. Visit $\mathrm{http} / / / \mathrm{www}$.dovepress.com/testimonials.php to read real quotes from published authors. 